\title{
VLBI astrometry toward Sgr D HII region with VERA
}

\section{Daisuke Sakai ${ }^{1,2}$, Tomoaki Oyama ${ }^{2}$, Takumi Nagayama ${ }^{2}$, Mareki Honma ${ }^{2,3,4}$ and Hideyuki Kobayashi ${ }^{1,3}$}

\author{
${ }^{1}$ Department of Astronomy, Graduate School of Science, The University of Tokyo, 7-3-1 \\ Hongo, Bunkyo-ku, Tokyo 113-0033, Japan \\ ${ }^{2}$ Mizusawa VLBI Observatory, National Astronomical Observatory of Japan, 2-12 \\ Hoshi-ga-oka, Mizusawa-ku, Oshu-shi, Iwate 023-0861, Japan \\ ${ }^{3}$ Mizusawa VLBI Observatory, National Astronomical Observatory of Japan, 2-21-1, Osawa, \\ Mitaka, Tokyo 181-8588, Japan \\ ${ }^{4}$ Department of Astronomical Sciences, Graduate University for Advanced Studies, 2-21-1, \\ Osawa, Mitaka, Tokyo 181-8588, Japan
}

\begin{abstract}
The Galactic centre region shows outstanding non-circular motion unlike the Galactic disk. As scenarios describing this non-circular motion, resonance orbits formed by the Galactic bar potential or expanding motion by past activity of the central $\mathrm{BH}$ are proposed. However, these both scenarios are based on line-of-sight velocities of molecular clouds in this region, and such one-dimension velocity information is insufficient to separate these scenarios.

To reveal dynamics of the Galactic centre region, we conducted astromertic observations of $22 \mathrm{GHz}$ water maser sources toward the Galactic centre direction. We conducted astrometric observations toward water maser source associated with Sgr D HII region. As a result, we succeeded to measure the parallax and proper motion of the maser source. The measured distance was $2.36(+0.58 /-0.39) \mathrm{kpc}$. This result clearly indicates that this source is not associated with the Galactic centre, but located on the Galactic disk.
\end{abstract}

Keywords. Galaxy: kinematics and dynamics, Masers, Astrometry

\section{Introduction}

Sgr D region is one of the largest radio continuum sources toward the Galactic center region, which is discovered in early radio observations (Downes \& Maxwell(1966)). This source mainly consists of Sgr D HII region (G1.13-0.10) on the northern part and Sgr D SNR region (G1.05-0.15) on the southern part. However, recent X-ray observations with Suzaku discovered another SNR behind the HII region (Sawada et al.(2009)). They argued that the new SNR is located in or behind the Galactic center because the X-ray absorption feature was detected against giant molecular cloud (GMC) in the Galactic center region, whose radial velocity is $\sim+100 \mathrm{~km} \mathrm{~s}^{-1}$.

Although various kind of observations suggested the distance to the Sgr D complex, consistent agreement has not been obtained. In fact, Sgr D HII region, Sgr D SNR region, and a new SNR detected with Suzaku are probably just a chance projection, and thus these distances are different with each other. From line-of-sight velocity of CS $J=1-0$ molecular line emission and $\mathrm{H} 91 \alpha$ radio recombination line emission at $V_{\mathrm{lsr}}=-20 \mathrm{~km} \mathrm{~s}^{-1}$, some literatures with early observations locate Sgr D on the Galctic center which has much more complex dynamics than the disk (Lis(1991)). On the other hand, narrow line width of the CS emission $\left(3.7 \mathrm{~km} \mathrm{~s}^{-1}\right)$ is unlikely to be in the Galactic center region because GMCs in the Galactic center region generally have large velocity dispersion $\left(10-20 \mathrm{~km} \mathrm{~s}^{-1}\right.$ FWHM) (Güsten(1989)). 

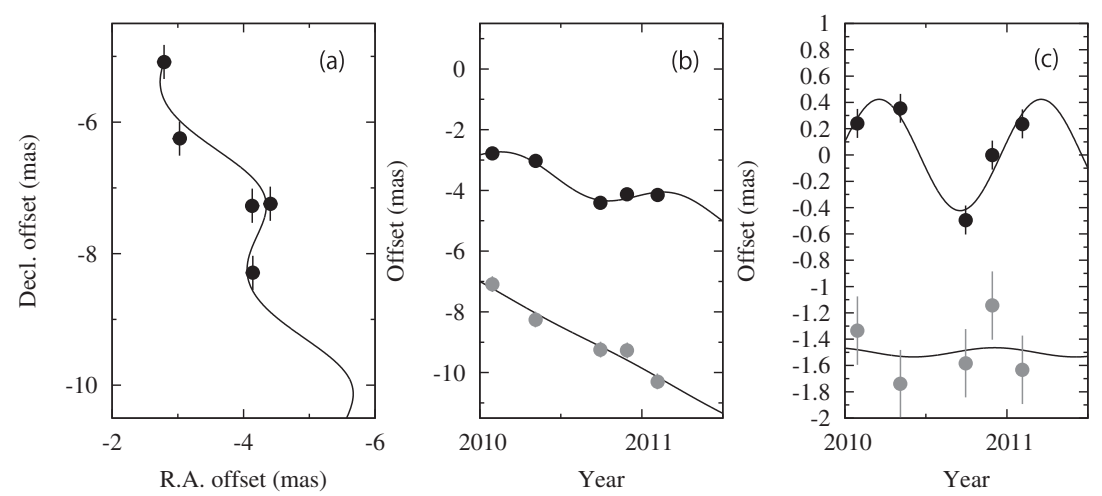

Figure 1. (a) Absolute motions of maser feature A5 in Sgr D HII region. Filled circles show the observed points from phase referencing. (b) Motions toward R.A. and Dec. as a function of time. Black circles show the motion toward R.A. direction, and gray circles show the motion toward Dec. direction. (c) Result of parallax fitting. Error bars are evaluated so that a $\chi^{2}$ value in the model fitting becomes to unity.

$22 \mathrm{GHz} \mathrm{H}_{2} \mathrm{O}$ maser emission has been detected toward Sgr D HII region with velocity of $-20 \mathrm{~km} \mathrm{~s}^{-1}$ (Mehringer et al.(1998)). It suggests that this water maser emission is also associated with Sgr D HII region.

\section{Results}

From phase-reference observations, we measured parallax and absolute proper motions relative to an extragalactic source J1745-2820. Figure 1(a) shows the projected motion of the maser spot. The direction of absolute proper motion is from north-east to south-west direction. This is almost parallel to the Galactic plane. In Figure 1(b), the motion toward R.A. and Dec. directions are shown as a function of time. By subtracting expected linear motions from Figure 1(b), we got parallax information as shown in Figure 1(c).

The absolute proper motions toward R.A. and Dec. are $-1.32 \pm 0.15 \mathrm{mas} \mathrm{yr}^{-1}$ and $-2.86 \pm 0.34$ mas $^{-1} r^{-1}$ respectively. The large error on Declination would be due to low elevation of the source. The obtained parallax value is $0.423 \pm 0.083$ mas. This is corresponding to $d=2.36_{-0.39}^{+0.58} \mathrm{kpc}$ in distance domain. The error bars of Figure 1 are 0.11 mas in right ascension and 0.26 mas in declination, respectively. This is derived by setting $\chi^{2}$ per degree to be unity for both the right ascension and the declination data.

This distance is corresponding to the Scutum arm. Because the velocities of water maser, CS molecular line, and H91 $\alpha$ radio recombination line are similar (around $-16 \mathrm{~km} \mathrm{~s}^{-1}$ ), the water maser source is likely to associating with Sgr D HII region seen in radio continuum emission.

\section{References}

Downes, D. \& Maxwell, A. 1966, ApJ, 146, 653

Güsten, R. 1989, The Center of the Galaxy, 136, 89

Lis, D. C. 1991, ApJL, 379, L53

Mehringer, D. M., Goss, W. M., Lis, D. C., Palmer, P., \& Menten, K. M. 1998, ApJ, 493, 274

Sawada, M., Tsujimoto, M., Koyama, K., et al. 2009, PASJ, 61, 209 